\title{
A genetic analysis of locomotor activity in Drosophila melanogaster
}

\author{
Barrie Burnet, ${ }^{*}$ \\ Lynn Burnet,* \\ Kevin Connolly $\dagger$ and \\ Nigel Williamson*
}

\author{
* Department of Genetics, University of Sheffield, \\ Sheffield S10 2TN. \\ $\dagger$ Department of Psychology, University of Sheffield, \\ Sheffield S10 2TN.
}

The locomotor activity of flies observed as isolated individuals in an "open field" observation chamber contains two components-the amount and speed of movement. These are distinct behavioural phenotypes. The reactivity of flies to handling disturbance is reflected chiefly by changes in the speed rather than in the amount of locomotion, but there are significant differences in the overall pattern of expression of reactivity between males and females. The amount and speed of locomotion are under independent genetic control, but genes affecting movement in the "open field" may also have effects on the locomotor activity of virgin females when they are courted. Variation in the locomotor activity of females has little effect on the amount of wing vibration which males direct at them, but significantly affects the amount of contact stimulation by licking which they receive.

\section{INTRODUCTION}

The locomotor activity of adult Drosophila is an important factor affecting dispersal, the search for feeding and breeding sites, and avoidance of predation. At a more subtle level, it is an important determinant of mating success. That individual differences in activity are substantially under genetic control is already well established (Ewing, 1963; Connolly, 1963; Hay, 1972; Angus, 1973; van Dijken and Scharloo, 1979a; van Dijken et al., 1979). There are some difficulties in interpreting what the measured activity represents in these investigations which differ in the methods of observation employed, because no distinction has been made between the amount and speed of locomotor activity. Connolly (1967) distinguished between spontaneous activity and reactivity. Reactivity may be defined in terms of the change in level of measured activity over time following the disturbance of an individual on transfer to a constant environment. Spontaneous activity corresponds to the asymptotic level of expression of the animal as a steady state is reached. The responses to selection observed by Connolly $(1966,1967)$ indicate that spontaneous activity and reactivity are probably controlled by different genetic systems.
Individual differences in locomotor activity and reactivity have consequences in relation to sexual selection. Bastock (1956) observed that more active females appeared to receive less courtship stimulation. Evidence that there is a relationship between locomotor activity and mating success is that responses to selection for an increased level of expression of one character may be accompanied by correlated change in the other (Manning, 1961, 1963; Burnet and Connolly, 1974). Van Dijken and Scharloo (1976b) found that females preferred high activity males. However, the effects of differences in locomotor activity also depend on which sex is being considered. Hyperactive females may also have an abnormally high threshold of sexual receptivity (Burnet and Connolly, 1974). As Manning (1961) pointed out: "Natural selection will normally lead to levels of activity and sexual behaviour being positively correlated and at an optimum which does not result in over-responsiveness in either direction". However, artificial selection, or close inbreeding to produce isogenic lines, could upset the genetic organisation underlying such an adaptive optimum.

Here we provide a description of locomotor activity as it is expressed in individuals of an adapted wild population making a distinction 
between the amount and speed of locomotor activity. Using inbred lines we then examine the genetic basis of these measures, and assess the interactive effects of variation in female locomotor activity during courtship.

\section{MATERIALS AND METHODS}

\section{Stocks and culture}

The Oregon and Formosa stocks are isogenic inbred strains maintained for many generations under a constant schedule of brother-sister mating. Eight derived inbred strains representing all possible homozygous combinations of the three major chromosomes from the Oregon and Formosa stocks were synthesised by using a 10 generation crossing scheme with the marked inversion containing balancer chromosomes $\operatorname{In}(2 L R) S M 5$, $a l^{2} C y 1 t^{v} \mathrm{cn}^{2} s p^{2} \quad$ and $\quad \operatorname{In}(3 L R) T M 3$, $y^{+}$rip $^{p}$ sep $b x^{34 e} e^{s}$ and the marked chromosomes $B 1 L$ and $S b$. Descriptions of balancer chromosomes and marker genes are given by Lindsley and Grell (1968). For each of the eight derived strains the designation of chromosome 1 refers to the origin of both the $\mathrm{X}$ and the $\mathrm{Y}$ as appropriate. The dot-like fourth chromosome was uncontrolled.

The Sierra Leone wild type laboratory population was founded from a sample of 30 isofemale lines each derived from a different gravid female in a transitional habitat at Freetown, Sierra Leone, West Africa. The cage population was maintained at $25^{\circ} \mathrm{C}$ in a large glass tank $(6.75$ cubic feet $)$ to which 20 half-pint food containers were added weekly. Each set of containers was left in the cage for three weeks so that 60 containers were always present in the cage at any one time. The population density was in excess of 5000 adult flies. Watch glasses, with 2 per cent agar gel containing 2 per cent ethanol and 1 per cent acetic acid, and overlaid by a thin layer of fresh live baker's yeast were placed in the cage. After $20 \mathrm{~min}$ the watch glasses were removed and each placed in a covered petri dish. Newly-hatched first instar larvae were transferred at a constant density of 50 larvae per culture to agar-oatmeal-treacle medium seeded with fresh live baker's yeast.

Virgin flies were lightly anaesthetised and separated within 12 hours after eclosion from the pupae and kept in single sex groups transferred daily to fresh live yeast medium. No further anaesthesia of any kind was used subsequent to separation of the virgin flies. All flies were cultured at $25 \pm 0.5^{\circ} \mathrm{C}$ under constant humidity on a constant light/dark cycle.
All behavioural observations were made in the same constant temperature room in which the stocks were reared and during the period from $1 \mathrm{~h}$ to $5 \mathrm{~h}$ after the flies' dawn.

\section{Measurement of locomotor activity}

A single larva aged 72 hours after hatching from the egg was placed onto fresh live baker's yeast layered over 3 per cent agar gel in a $10 \mathrm{~cm}$ diameter petri dish. After a $1 \mathrm{~min}$ settling down period the number of body contractions was recorded in each successive minute for five consecutive min.

The locomotor activity of adult flies was observed using a $10 \times 10 \times 1 \mathrm{~cm}$ deep chamber made from white perspex and fitted with a clear perspex lid engraved with a grid of $1 \mathrm{~cm}$ squares. A single fly was aspirated into the chamber through an entry port which was then closed by a perspex plug. The observation chamber was then given a single smart tap and the activity of the fly observed for $10 \mathrm{~min}$. Using a multichannel event recorder the amount of time spent in locomotion, preening, and standing was recorded in each successive minute together with the number of jumps and the number of $1 \mathrm{~cm}$ squares entered whilst the fly was engaged in locomotion. Speed of locomotion, expressed as centimetres per second of movement is adjusted for differences in the amount of time spent in locomotion in different minutes. The Sierra Leone flies were all 5-day-old virgins. The activity of flies from the eight inbred lines derived by chromosome substitution was observed using the same procedure except that the chamber was not tapped. Each fly was allowed a settling down period of $2 \mathrm{~min}$ after which the amount and speed of locomotion was recorded for the next $100 \mathrm{~s}$. These flies were all 3-day-old virgins.

\section{Courtship behaviour}

Courtship of a single pair of 3-day-old virgin flies was observed in a circular observation chamber $19 \mathrm{~mm}$ in diameter and $7 \mathrm{~mm}$ deep made of white perspex and fitted with a clear perspex lid. The flies were observed using a double-headed binocular microscope so that two observers could record simultaneously using different input channels of the same multichannel event recorder. Observations were made from the start of courtship, defined as the beginning of the first bout of wing vibration until copulation, or for a total observation period of $1000 \mathrm{~s}$. Locomotor activity during courtship was measured as the proportion of time spent in locomotion whilst the male was 
oriented to the female, i.e., whilst the male was courting. The proportion of orientation time spent in wing vibration, and the number of licks and attempts to effect genital engagement by the male were recorded. Licking and genital contact were recorded only when actual contact between the labellum or claspers, of the male with the genitalia of the female was observed to occur. Near misses were not included in the analyses.

\section{Statistical procedures}

The three-way Analysis of Variance procedure used for the analysis of chromosome main effects and interactions in table 3 is described by Sokal and Rohlf (1981). The raw scores for the proportions of time spent in locomotion in the open field and in courtship were transformed to the arcsine (radians). A proportional relationship between the means and standard deviations for the speed scores within the eight derived chromosome substitution lines necessitated a log transformation to achieve variance stability. The raw scores from the Sierra Leone population for proportions of time spent in locomotion and preening were transformed to the arcsine (degrees).

\section{RESULTS}

\section{Activity of Sierra Leone wild flies}

After entering the observation chamber the locomotor activity of Sierra Leone adult flies consisted of running, walking and jumping. The height of the chamber constrained flight activity but flights did occasionally occur. When not engaged in locomotion the fly stood still or preened. Locomotion and preening are mutually exclusive activities. Fig. 1 shows the amount of time spent in locomotion or preening, measured as a proportion of each successive minute. The amount of locomotion was consistently higher in adult females than in males, and the regression constants in table 1 estimating the initial amount of locomotor activity in the two sexes do not differ significantly. The regression coefficients, which describe the amount of activity over time, show that for males there was a significant reduction in the amount of locomotion with time whereas for females it remained unchanged.

Initially the males moved much faster than the females, and both sexes showed a decline in speed of locomotion until the mean scores were about the same after $10 \mathrm{~min}$ (fig. 2). After entering the observation chamber the flies tended to jump (fig.

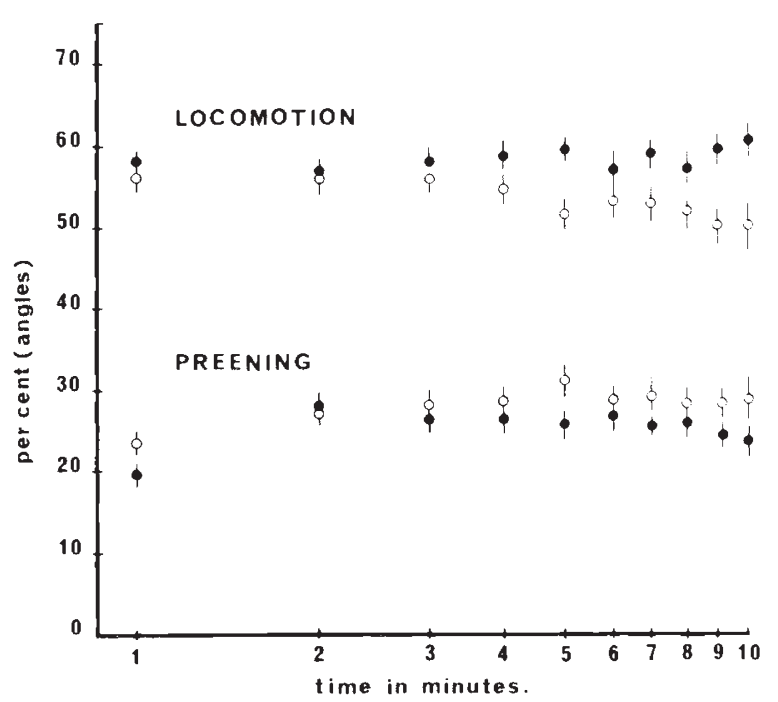

Figure 1 The amount of locomotor activity and preening in the open field for adult flies from the Sierra Leone population expressed as the mean \pm S.E. (arcsine) proportion of time spent per minute. Each point is based on observations from 180 individuals. females, $\bigcirc$ males.

3). Females tended to jump more than males, but the rate at which jumping occurred declined rapidly with time.

The high levels of expression of speed of locomotion and jumping observed immediately after disturbance on entering the observation chamber, and their subsequent decay to a lower level of expression over a period of minutes, reflects the reactivity of the flies. Evidently the rate at which reactivity decays depends upon the measure through which it is expressed, since the change to a "settled" level is quicker in terms of jumping than for speed of movement. Moreover the sexes differed quite strikingly in the way in which reactivity was expressed. Males were clearly more reactive than females in terms of speed of movement. Males also exhibited reactivity in terms of amount of movement whereas the females did not (fig. 1).

Table 1 Regression constants and slope coefficients for change in the amount and speed of locomotion in the open field over $\log$ time shown in Figs 1 and 2

\begin{tabular}{lcrl}
\hline & \multicolumn{1}{c}{ Constant } & Coefficient & $P$ \\
\hline Amount of locomotion & & & \\
Female & $57.29 \pm 0.93$ & $1.846 \pm 1.28$ & ns \\
Male & $57.43 \pm 0.94$ & $-6.317 \pm 1.30$ & $<0.01$ \\
Speed & & & \\
Female & $1.141 \pm 0.013$ & $-0.167 \pm 0.017$ & $<0.001$ \\
Male & $1.531 \pm 0.017$ & $-0.529 \pm 0.023$ & $<0.001$ \\
\hline
\end{tabular}




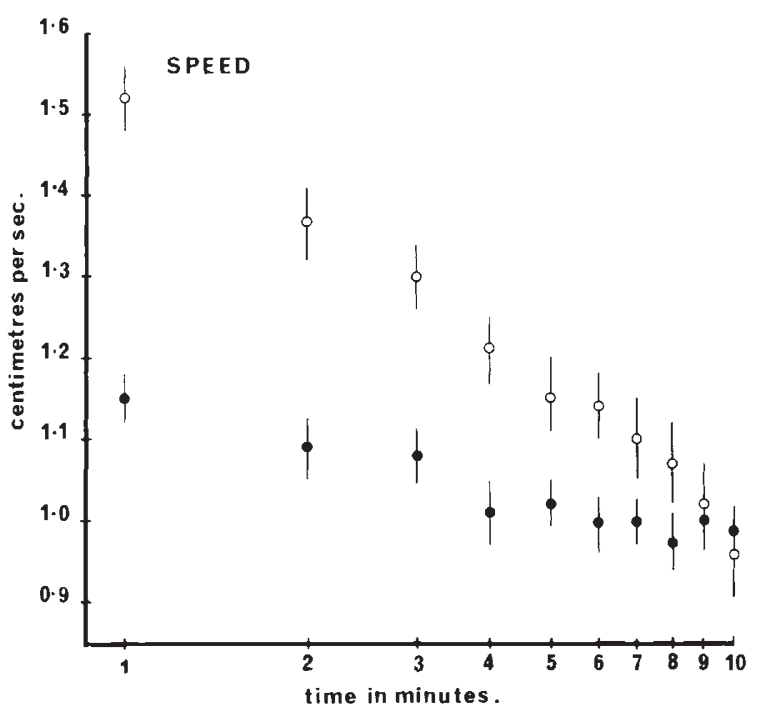

Figure 2 Speed of movement for adult flies of the Sierra Leone population expressed as the mean \pm S.E. centimetres per second. females, $\bigcirc$ males.

The proportion of time spent preening by both sexes was low in the first minute of observation and increased to a maximum after two minutes. Preening by males then stayed at a constant level during the rest of the observation period, whereas females showed a slow but significant decline in preening $(P<0 \cdot 001)$ after the second minute. Change in preening behaviour affords another indication of reactivity.

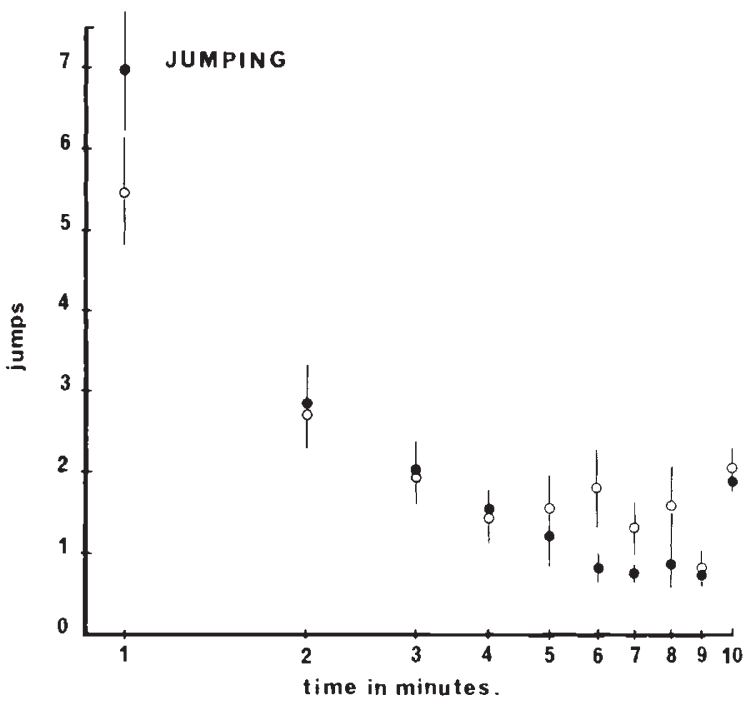

Figure 3 Mean \pm S.E. number of jumps per minute by adult flies of the Sierra Leone population. females, $O$ males.

\section{Correlations between measures}

The correlations between behaviours in males and females for which measures of both larval and adult activity were available are shown in table 2 . The locomotor activity of larvae involves a wave of contraction and extension which proceeds anteriorly from the anal segments and terminates at the head. The passage of one such wave constitutes a unit of larval locomotion (Green et al., 1983). When measured in terms of the number of waves per minute the rate of larval locomotion has the advantage of being independent of differences in larval size, which is not so for measures based on the linear distance they travel (Godoy-Herrera et al., 1984).

Correlations for the adult flies were computed using the means of the transformed scores taken over the whole $10 \mathrm{~min}$ observation period.

There was no significant correlation within individuals between the rate of larval locomotion and the amount, or speed, of adult locomotor activity, suggesting that the underlying organisation of genetic control is separate at these two stages in the life history. In males there was a significant negative correlation between the rate of larval movement and the proportion of time spent preening at the adult stage, but there was no significant association between these variables in females.

A fly preens only when standing still, so a strong negative correlation between the amounts of adult locomotion and preening is expected. Positive correlations, significant in both sexes, between the speed and the amount of locomotion indicate that the fastest moving individuals are those which move about most and also jump significantly more often.

Table 2 Correlations between measures of larval and adult activity for males and females of the Sierra Leone population $(N=50$ for each sex)

\begin{tabular}{|c|c|c|c|c|}
\hline \multirow{2}{*}{$\begin{array}{l}\text { Adult } \\
\text { activity }\end{array}$} & & \multirow{2}{*}{$\begin{array}{l}\text { Larval } \\
\text { locomotion }\end{array}$} & \multicolumn{2}{|c|}{ Adult activity } \\
\hline & & & Jumping & Preening Locomotion \\
\hline Speed & $\begin{array}{l}q \\
0 \\
0\end{array}$ & $\begin{array}{l}0 \cdot 169 \\
0 \cdot 114\end{array}$ & $\begin{array}{l}0 \cdot 299^{*} \\
0 \cdot 389^{* *}\end{array}$ & $\begin{array}{l}-0.078 \quad 0.439^{* *} \\
-0.491^{* *} 0.433^{* *}\end{array}$ \\
\hline Locomotion & $\begin{array}{l}q \\
\delta\end{array}$ & $\begin{array}{l}0 \cdot 150 \\
0 \cdot 241\end{array}$ & $\begin{array}{l}-0.005 \\
-0.158\end{array}$ & $\begin{array}{l}-0.719^{* *} \\
-0.888^{* *}\end{array}$ \\
\hline Preening & $\begin{array}{l}q \\
0\end{array}$ & $\begin{array}{l}-0.065 \\
-0.293^{*}\end{array}$ & $\begin{array}{l}-0.036 \\
-0.071\end{array}$ & \\
\hline Jumping & & $\begin{array}{l}0.193 \\
0.252\end{array}$ & & \\
\hline
\end{tabular}

$*$ and $* *$ denote correlation coefficients significant at the 5 and 1 per cent level of probability, respectively. 
Genetic analysis by chromosome exchange

If amount and speed are distinct but related aspects of the expression of locomotor activity in the open field, as the temporal differences in their level of expression in the Sierra Leone population suggest, this implies that there are also differences in their underlying genetic control. A comparison of the genetic structures was made using two isogenic inbred laboratory strains, Oregon and Formosa, which differ significantly in locomotor activity. Marked inversion containing balancer chromosomes were used to derive the eight strains containing all possible homozygous combinations of the three major chromosomes of the parent strains. The strain means for amount and speed of open field locomotor activity of virgin females from each of the derived stocks is shown in table 3. A threeway analysis of variance was used to test for chromosome main effects and interactions.

The amount of locomotion was significantly affected by differences in chromosome 3 alone, whereas speed was significantly affected by differences in chromosomes 1 and 2 as well as by chromosomal interactions. Evidently the differences in the amount and speed of locomotor activity between these strains are controlled by different genes.

Fig. 4 shows the amount of locomotor activity exhibited by females of each of the derived stocks during courtship. This was measured as the mean proportion of courtship time the female spent moving whilst the male was oriented to her, and does not include locomotion during breaks in courtship. Oregon males from the isogenic inbred parent stock were used throughout so that there is no variation in male genotype. Females of the different genotype groups differ significantly in the amount of locomotor activity during courtship. The strain means are shown in table 3 .

Three-way analysis of variance shows that the differences in the amount of locomotion in courtship include significant main effects of chromosomes 1 and 3 as well as significant interactions between all three major chromosomes. Genetic control of the amount of locomotion in courtship

Table 3 Chromosome substitution analysis of the amount of female locomotor activity in the open field, and in courtship. The amount is the mean (arcsine) proportion of time spent moving. Speed is the mean $(\log )$ centimetres per second. Locomotor activity in courtship is time spent moving by the female whilst the male was oriented. The lower part of the table shows the magnitude and direction of significant deviations from the grand mean $\mathrm{G}$ over all genotype groups caused by homozygous replacement of Oregon by Formosa chromosomes. Each mean \pm S.E. is based on observations from 50 females

\begin{tabular}{|c|c|c|c|c|c|}
\hline \multicolumn{3}{|c|}{ Chromosome } & \multicolumn{2}{|l|}{ Open field } & \multirow[b]{2}{*}{ In courtship } \\
\hline 1 & 2 & 3 & Amount & Speed & \\
\hline 0 & 0 & 0 & $0.6817 \pm 0.0605$ & $0.07451 \pm 0.00235$ & $0.6546 \pm 0.0439$ \\
\hline $\mathrm{F}$ & 0 & 0 & $0.6826 \pm 0.0504$ & $0.06558 \pm 0.00339$ & $0.3960 \pm 0.0367$ \\
\hline 0 & $\mathrm{~F}$ & 0 & $0.6255 \pm 0.0604$ & $0.07906 \pm 0.00169$ & $0.4868 \pm 0.0402$ \\
\hline 0 & 0 & $\mathrm{~F}$ & $0.8195 \pm 0.0509$ & $0.08281 \pm 0.00186$ & $0 \cdot 8408 \pm 0 \cdot 3467$ \\
\hline $\mathrm{F}$ & $\mathrm{F}$ & 0 & $0.5898 \pm 0.0526$ & $0.07866 \pm 0.00207$ & $0.6312 \pm 0.0375$ \\
\hline $\mathrm{F}$ & 0 & $F$ & $0.7919 \pm 0.0504$ & $0.06890 \pm 0.00262$ & $0.5167 \pm 0.0384$ \\
\hline 0 & $\mathrm{~F}$ & $F$ & $0.7727 \pm 0.0526$ & $0.07711 \pm 0.00306$ & $0.6588 \pm 0.0409$ \\
\hline$F$ & $\mathrm{~F}$ & $F$ & $0.7866 \pm 0.0682$ & $0.07169 \pm 0.00298$ & $0.5147 \pm 0.0357$ \\
\hline G & & & 0.7188 & 0.07479 & 0.5875 \\
\hline \multicolumn{6}{|c|}{ Chromosome } \\
\hline 1 & & & & $-0.00358^{* * *}$ & $-0.0727^{* * *}$ \\
\hline 2 & & & & $+0.00184^{*}$ & \\
\hline 3 & & & $+0.0739 * * *$ & & $-0.0454^{* * *}$ \\
\hline \multicolumn{6}{|c|}{ Interactions } \\
\hline $1 \times$ & & & & $+0.00213^{*}$ & $+0.0729^{* * *}$ \\
\hline $1 \times$ & & & & & $-0.0442^{* * *}$ \\
\hline $2 \times$ & & & & $-0.00256^{* *}$ & $-0.0314^{*}$ \\
\hline & & & & & $-0.0281^{*}$ \\
\hline
\end{tabular}

* ** and *** denote deviations which are significant at the 5,1 and $0 \cdot 1$ per cent levels of probability, respectively. 


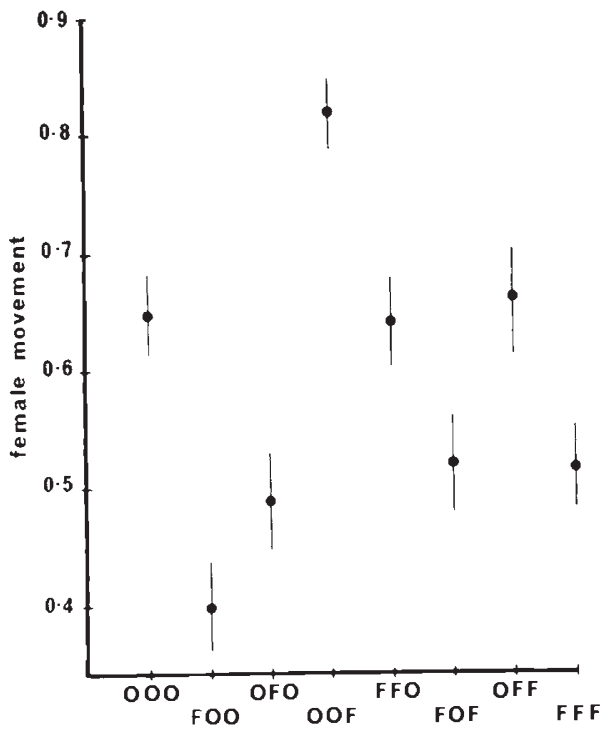

Figure 4 Amount of time spent moving by females in courtship in eight genotype groups representing all possible homozygous combinations of the three large chromosomes derived from the Oregon and Formosa isogenic lines. Female movement is time spent moving whilst the male was oriented (mean \pm S.E. arcsine). Each point is based on observations for 50 females.

is clearly more complicated than in the open field but the direction of the third chromosome effect is the same in both situations.

Another interesting feature of the results summarised in table 3 is that across genotype groups there is a significant correlation $(r=0.78 P<0.05)$ between amount of female locomotor activity in courtship and the speed of open field locomotion. The direction of the significant chromosome 1 effect, and the interactions between chromosomes $1 \times 2$ and $2 \times 3$ are the same for both of these characters.

\section{Locomotor activity and male generated courtship stimuli}

During courtship the male orients so that his body axis points directly towards the female. If she moves he follows, so that male locomotor activity during courtship is, to a great extent, dependent upon that of the female. Males frequently vibrate a single wing whilst following a moving female. One of the effects of the acoustic stimuli generated by wing vibration is to induce the female to slow down or become stationary. Fig. 5 shows that the proportion of courtship time spent in wing vibration by Oregon males was about the same in pair-

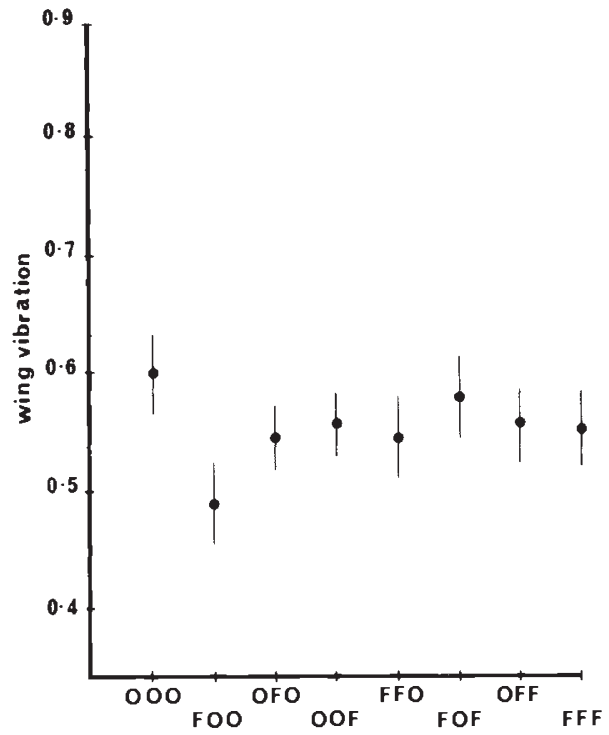

Figure 5 The proportion of orientation time spent vibrating by Oregon males courting females of different genotype groups. Wing vibration is expressed as the mean \pm S.E. (arcsine) and based on observations for 20 courtships.

ings with females of genotypes which differed significantly in their mean time spent moving. The correlation within genotype groups between female locomotion and amount of male wing vibration was positive in seven out of the eight groups, but significant in only one of them (table 4).

Whilst locomotor activity would seem unlikely to have had a significant effect on the amount of acoustic stimulation received by females it did affect the contact stimuli which they received. Fig. 6 shows that the mean number of licks per second of orientation received by females of different

Table 4 Intraclass correlations within genotype groups between locomotor activity by the female during courtship and wing vibration, licking, and attempted copulation by Oregon males

\begin{tabular}{|c|c|c|c|c|c|}
\hline \multicolumn{3}{|c|}{$\begin{array}{l}\text { Female } \\
\text { genotype }\end{array}$} & \multirow{2}{*}{$\frac{\text { Vibration }}{+0.429}$} & \multirow{2}{*}{$\begin{array}{l}\text { Licking } \\
-0 \cdot 327^{*}\end{array}$} & \multirow{2}{*}{$\begin{array}{c}\begin{array}{c}\text { Attempted } \\
\text { copulation }\end{array} \\
-0.326^{*}\end{array}$} \\
\hline 0 & 0 & 0 & & & \\
\hline $\mathrm{F}$ & 0 & 0 & $+0.539^{*}$ & $-0 \cdot 670^{* * *}$ & $-0 \cdot 302^{*}$ \\
\hline 0 & $\mathrm{~F}$ & 0 & +0.349 & $-0 \cdot 242$ & $-0.559 * * *$ \\
\hline 0 & 0 & $\mathrm{~F}$ & +0.408 & $-0 \cdot 540^{* * *}$ & $-0 \cdot 105$ \\
\hline $\mathrm{F}$ & $\mathrm{F}$ & 0 & +0.376 & $-0 \cdot 503^{* * *}$ & -0.265 \\
\hline $\mathrm{F}$ & 0 & $\mathrm{~F}$ & +0.434 & $-0 \cdot 570^{* * *}$ & $-0 \cdot 170$ \\
\hline 0 & $\mathrm{~F}$ & $\mathrm{~F}$ & $-0 \cdot 011$ & $-0 \cdot 554^{* * *}$ & $-0 \cdot 373^{* *}$ \\
\hline$F$ & $F$ & $F$ & $+0 \cdot 298$ & $-0 \cdot 490^{* * *}$ & $+0 \cdot 021$ \\
\hline
\end{tabular}

* and $* * *$ denote correlation coefficients which are significant at the 5 and $0 \cdot 1$ per cent level of probability, respectively. 


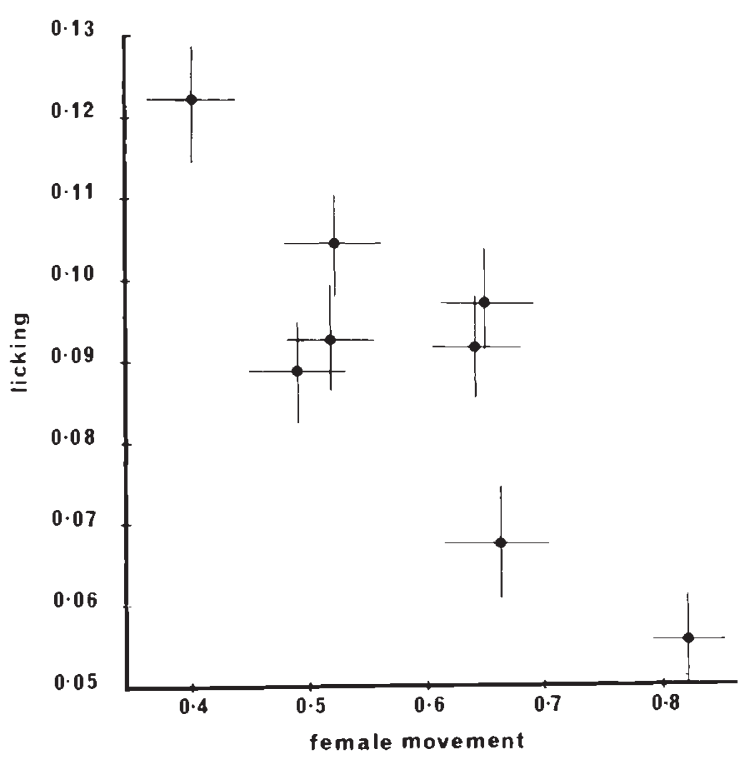

Figure 6 The relationship between the amount of locomotor activity by females of different genotype groups and the number of licks per second of orientation by Oregon males. Each point is based on observations for 50 pairs.

genotype groups was inversely related to the proportion of time they spent moving. The correlations between licking and movement within groups were negative, and significantly so in seven out of the eight groups (table 4). The proportion of licks followed by genital contact in attempted copulation was inversely related to the amount of female locomotor activity in seven out of the eight genotype groups. This trend was statistically significant in four of them (table 4).

Although the amount of wing vibration performed by males is not significantly affected by the locomotor activity of the females to which it is directed (fig. 5), there are highly significant differences in the amount of wing vibration provided by males of different genotype groups when compared across matings with inbred Oregon females. The proportion of orientation spent vibrating by 50 males from each of the eight homozygous combinations of $\mathrm{O}$ and $\mathrm{F}$ chromosomes was compared with the amount of locomotor activity shown by their sisters, in the same number of pairings with Oregon inbred males (table 3). Consequently, differences between genotypes for a given sex were always compared using standard inbred Oregon individuals of the opposite sex. The relationship is shown in fig. 7 . What is interesting here is that females from genotype groups with a high mean level of locomotor activity in courtship have brothers

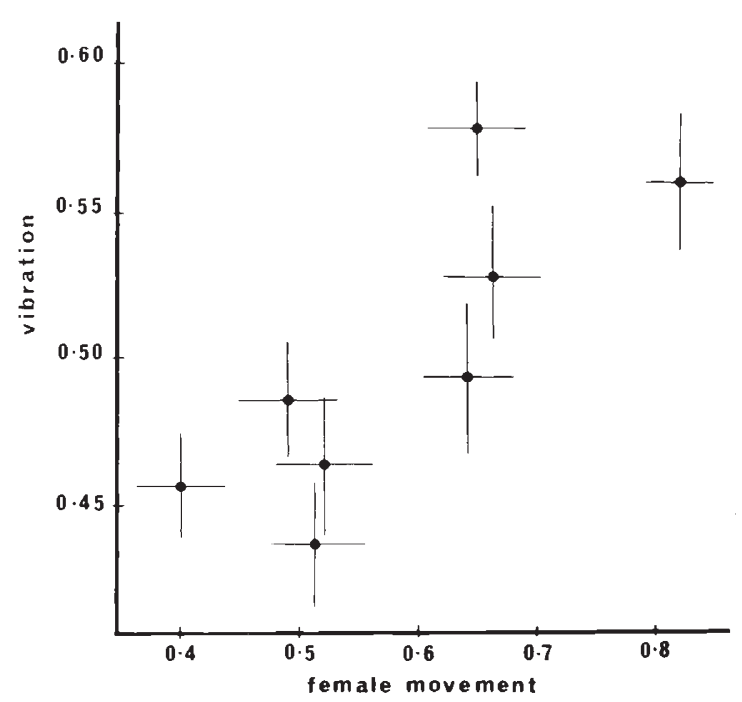

Figure 7 Relationship between the locomotor activity of females of different genotype groups courted by Oregon males and the amount of wing vibration by males of the same genotype groups when courting Oregon females.

which provide a large amount of wing vibration. There is evidence here for a genetic correlation between locomotor activity in females and the intensity of wing vibration stimuli generated by males.

\section{DISCUSSION}

Partition of locomotor activity of adult Drosophila into separate measures of amount and speed shows that reactivity is detectable more as a change in the speed rather than the amount of locomotion in the Sierra Leone population. The flies respond to disturbance by jumping, increase in speed, and by reduced preening, and these associated changes in behaviour may together constitute a "startle response" which is likely to be adaptive in nature.

Hay (1972) and Angus (1974) used a time sampling technique to study the locomotor activity and preening behaviour of flies after being disturbed, either by a mechanical or a shadow stimulus. As Sewell (1979) notes, there is some doubt as to whether apparently similar components of activity measured in this way are directly comparable with those measured in the "open field" as described here. With this caveat in mind, our results agree with those of Angus (1974) in showing a reduction in preening following disturbance. Both Hay (1972) and Angus (1974) found evidence of directional dominance for high activity which suggests a past history of selection for a high level of 
expression of the character (Mather, 1966). The measure of activity used is analogous to the amount of locomotor activity used in our study, but contains no information about the speed of locomotion.

Angus (1974) found that there was a reduction in activity, as well as in preening, following disturbance. Our results for the Sierra Leone population reveal little change in the amount of locomotor activity and that only in males. Angus does not show the results for the sexes separately, but it is likely that the apparent difference in detection of the expression of reactivity between the two studies arises either from differences in the strains used, or from the nature of the behavioural measures employed.

Chromosome substitution analysis shows that the amount and speed of locomotor activity are under the control of different genes. This does not, of course, prove that there are no genes which have effects on both the amount and speed of locomotion, only that such genetic differences as there are between the Oregon and Formosa strains used for our analysis involve differences at gene loci affecting the amount, or the speed of locomotion, but not both. The significant correlation between the amount and speed of locomotion within individuals of the Sierra Leone population may be a consequence of linkage relations between gene loci involved in the control of these separate systems but could also be due to the pleiotropic effects of certain genes affecting both measures. A comparison of the genetic architectures for amount and speed of locomotor activity should extend our understanding of the organisation of locomotor activity and reactivity.

Tompkins et al (1982) found that males must perceive female movement in order to initiate and perform normal courtship. Once courtship has begun the amount and speed of movement of the female may influence the rate and intensity of the courtship stimuli which she receives. Cook (1979), using a cybernetic approach, detected strain differences in the ability of courting males to track and maintain contact with moving females. Our results confirm that there is significant genetic variation in the amount of locomotor activity in courtship. Genes controlling the amount of movement in the open field, as well as those controlling the speed of locomotion, may also influence the amount of locomotion in courtship. The causal link here could be that these genes control female reactivity, and that this is the underlying parameter influencing both speed of locomotion in the open field and amount of movement in courtship.
The sequence analyses by Markow and Hanson (1981) showed that as courtship proceeds females spend less time in locomotion and more time in preening. Since an increase in preening activity also occurs when the flies are isolated, preening behaviour may have no specifically sexual connotation but does provide some indication of the level of female reactivity. Courtship reduces the activity of receptive females and this may be partly due to odour cues (Tompkins et al., 1982), and to the effects of acoustic stimuli generated through wing vibration by the male (Cook, 1973; von Schilcher, 1976). The amount of wing vibration appears to be relatively independent of differences in female locomotor activity, although it may not be so in situations involving females from selected lines, or for neurological mutants, which cause more extreme deviations in the expression of female mobility. Males attempt to lick moving as well as stationary females, but the amount of contact stimulation actually received by females is inversely related to their locomotor activity. As the male extends his proboscis to lick he grasps the abdomen of the female with his forelegs and simultaneously curls the tip of his abdomen down in preparation for genital contact. Markow and Hanson (1981) observed that successful copulation attempts are always directed at immobile females, and that slowing down is the only visible indication that $D$. melanogaster females are ready to accept genital contact. Contact stimulation of the female by licking seems to be important in inducing the stationarity necessary to allow genital engagement which precedes mounting by the male. The proportion of licks followed by genital contact also tends to be inversely related to the amount of female locomotion, but the relationship is weak, which implies that if the female receives contact stimulation from the male through licking then genital engagement usually follows. Evidently reduced levels of licking are likely to be an important determinant of the reduced mating success of hyperactive females (Manning, 1961; Burnet and Connolly, 1974).

\section{REFERENCES}

ANGUS, J. 1974. Genetic control of activity, preening, and the response to a shadow stimulus in Drosophila melanogaster. Behav. Genet., 4, 317-329.

BASTOCK, M. 1956. A gene mutation which changes a behaviour pattern. Evolution, 10, 421-439

BURNET. B. AND CONNOLLY, K. 1974. Activity and sexual behaviour in Drosophila melanogaster. In Abeelen, J. H. F. van (ed.) The Genetics of Behaviour, North-Holland Publishing Company, Amsterdam, Oxford, pp. 201-258. 
CONNOLLY, K. 1966. Locomotor activity in Drosophila. II, Selection for active and inactive strains. Anim. Behav., 14, 444-449.

CONNOLLY, K. 1967. Locomotor activity in Drosophila. III, A distinction between activity and reactivity. Anim. Behav., $15,149-152$.

COOK, R. M. 1973. Courtship processing in Drosophila melanogaster. II, An adaptation to selection for receptivity to wingless males. Anim. Behav., 21, 349-358.

COOK, R. M. 1979. The courtship tracking of Drosophila melanogaster. Biol. Cybernetics, 34, 91-106.

DIJKEN, F. R. VAN AND SCHARLOO, w. 1979a. Divergent selection on locomotor activity in Drosophila melanogaster. I, Selection response. Behav. Genet., 9, 543-553.

DIJKEN, F. R. VAN AND SCHARLOO, W. $1979 b$. Divergent selection on locomotor activity in Drosophila melanogaster. II, Test for reproductive isolation between selected lines. Behav. Genet., 9, 555-561.

DIJKEN, F. R. VAN, SAMBEEK, M. P. J. W. VAN AND SCHARLOO, W. 1979. Divergent selection on locomotor activity in Drosophila melanogaster. III, Genetic analysis. Behav. Genet., 9, 563-570.

EWING, A. W. 1963. Attempts to select for spontaneous activity in Drosophila melanogaster. Anim. Behav., 11, 369-378.

GODOY-HERRERA, R., BURNET, B., CONNOLLY, K. AND GOGARTY, J. 1984. The development of locomotor activity in Drosophila melanogaster larvae. Heredity, 52, 63-75.

GREEN, C. H., BUR NET, B. AND CONNOLLY, K. J. 1983. Organisation and patterns of inter- and intraspecific variation in the behaviour of Drosophila larvae. Anim. Behav., 31, 282291.
HAY, D. A. 1972. Genetical and maternal determinants of the activity and preening behaviour of Drosophila melanogaster reared in different environments. Heredity, 28, 311-336.

LINDSLEY, D. L. AND GRELL, E. H. 1968. Genetic Variations of Drosophila melanogster. Carnegie Institute of Washington. Publication 627.

MANNING, A. 1961. The effects of artificial selection for mating speed in Drosophila melanogaster. Anim. Behav., 9, 82-92.

MANNING, A. 1963. Selection for mating speed in Drosophila melanogaster based on the behaviour of one sex. Anim. Behav., 11, 116-120.

MARKOW, T. A. AND HANSON, S. J. 1981. Multivariate analysis of Drosophila courtship. Proc. Natl. Acad. Sci. U.S.A., 78, 430-434.

MAther, K. 1966. Variability and selection. Proc. Roy. Soc. B, $164,328-340$.

SCHILCHER, F. VON 1976. The function of pulse song and sine song in the courtship of Drosophila melanogaster. Anim. Behav., 24, 622-625.

SEWELL, D. F. 1979. Effect of temperature and density variation on locomotor activity in Drosophila melanogaster: A comparison of behavioural measures. Anim. Behav., 27, 312313.

SOKAL, R. R. AND ROHLF, F. J. 1981. Biometry, W. H. Freeman \& Co., New York.

TOMPKINS, L., GROSS, A. C., HALL, J. C., GAILEY, D. A. AND SIEGEL, R. W. 1982. The role of female movement in the sexual behaviour of Drosophila melanogaster. Behav. Genet., 12, 295-307. 